\title{
Measurement of Some Parameter in Wind Tunnel Experiment Based on ZigBee
}

\author{
Zhiqiang Yuan \\ Physical Science and Technology College of Yichun University, Yichun 336000, China
}

\begin{abstract}
The paper designed an energy-efficient, high-reliability wireless system for measuring torsion, revolution and pneumatic pressure in the wind tunnel testing of the wind turbine, based on the platform of the sensor network formed by all kinds of sensors and ZigBee nodes. The paper introduced the measuring method of sensors and the wireless method of the datum-transmitted, and provided the overall designed scheme, the hardware designed diagram of sensor nodes, the program flow diagram of sensornodes. It is verified that the testing result is much more than be hoped.
\end{abstract}

Keywords-zigbee; wireless sensor network; wind tunnel testing; wind turbine.

\section{INTRODUCTION}

Accompanied with development of the ecconomy, natural environment is seriously destroyed by high-pollution power plant such as coal-fired power-plant and photovoltaic power-plant. For the purpose of long-term benefit of the ecconomy and descendants, energy planning is urgent. Because exploit of hydroenergy and wind energy has the virture of low-pullution, reproducible, It is paid attention to by more country. Nowadays, the scale of wind power generation is already big, with the good prospects for development[1]. the preliminary design and test of wind power generation is completed in wind tunnel experiment of wind turbine[2], with the important para- meters of power performance, aerodynamic performance, vibration performance[3] [4]. The paper introduces a new method of measuring torque, speed and pressure based on relevance of power performance of wind turbine and torque, speed. Wireless method of measuring parameters of wind turbine is more popular because wired mesurement has the disadvantage of trouble-wiring and inconvenience to maintian [5]. The theoretical study of wireless communication network and mesurement[6] [7] [8] is a lot, most of which focusing on high-stability, wideband, multi-frequecy range. The ZigBee chip which is choosed by the paper is very suitable for constructing WSN, with its virture of low price, low power consumption and Ad Hoc network function[9].

\section{OVERALL DESIGN OF THE SYSTEM}

Overall design of the system includes sensor node, WSN and center computer, which is showed in Fig.1. The dotted arrowhead in the figure means wireless transmission. Lots of sensor nods formed by kinds of senors and ZigBee chip with signal transceiver mainly collect instantaneous experimental data. WSN is formed by lots of ZigBee nodes with Ad Hoc network function.ZigBee nodes can communicate with each other and automatically form network, waiting for exchang data at any time, finally the data being collected to the ZigBee router. Center computer connects ZigBee router by serial communiction mode with RS485 interface. Meanwhile there is a visuable software on the computer, which can observe instantaneous parameters data. When measuring parameters data go beyong rang of the setted value, center computer will automatically give an alarm and sending control signal to control unit by USB interface.

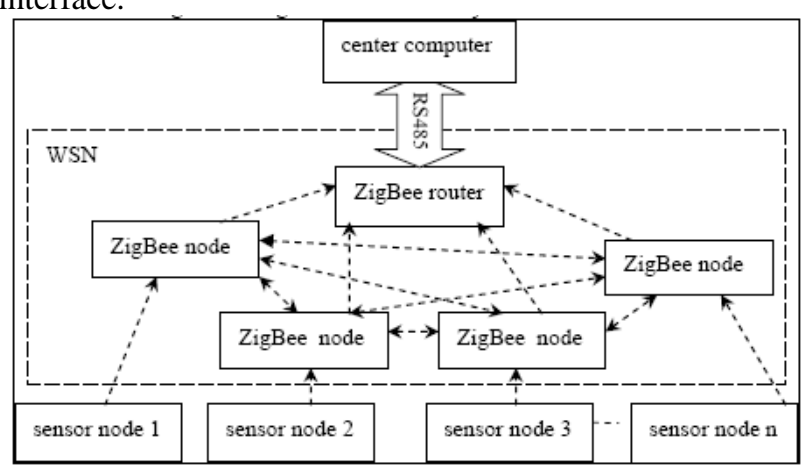

Figure 1. Overall design figure of the system

\section{HARDWARE DESIGN}

\section{A. Hardware design of sensor}

The sensor node in this design includes pneumatic-load node, temperature sensor node, torque and speed sensor. The paper analyzes the pneumatic pressure sensor node and torque-speed sensor node. The pneumatic pressure sensor node consists of ZigBee chip CC2530 and pneumatic pressure sensor. The chip MAS6512 made by MAS company is selected as the pneumatic pressure sensor. The chip can be used in I2C or SPI serial mode, with virture of low-voltage, low-power, low-noise. The pin 4 of the chip is connectted to positive supply. The pin 12 is connectted to ground, while the pin 5 and the pin 6 being connectted to SDA and SCL of CC2530. The hardware conneticon figure is showed in Fig.2. 


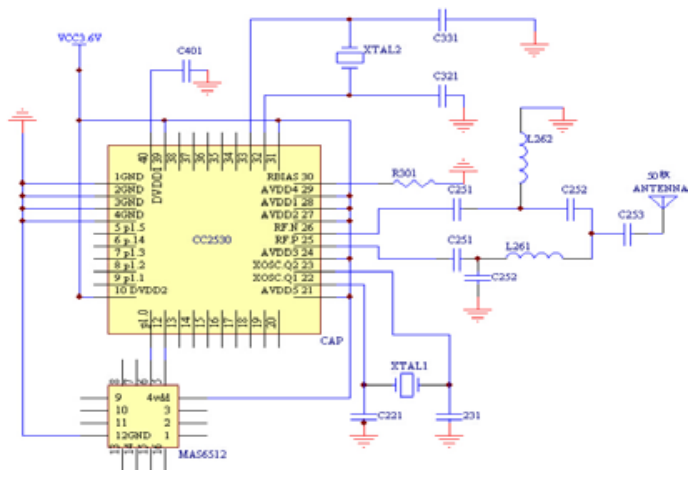

Figure 2. Hareware connection figure of sensor of pneumatic pressure

torque-speed sensor node consists of CC2530 and torque-speed sensor. ZH07 made in china is selected as torque-speed sensor, which has the virture of digital-output, real-time accurate measurement, srong anti-interference. The hardware connetion figure is showed in Fig.3.

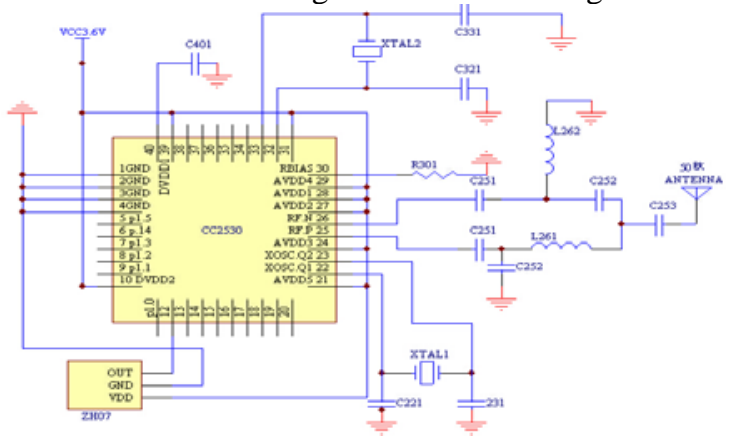

Figure 3. Hareware figure of sensor of the torque and speed

\section{B. Hardware design of ZigBee node and ZigBee router}

CC2530 is selected as ZigBee node, which is embeded with stong 8051 . CC2530 includes 256KB programmable flash, 8KB RAM, signal transceiver. Because ZigBee router process more signal than ZigBee node, CC2530 and MSP430G2553 made by TI company are selected as the ZigBee router. MSP430G2553 has the virture of fast calculation speed, ultra low power, abundant on-chip resources, which communicate with CC2530 by serial mode.

\section{SOFTWARE DESIGN}

CC2530ZDK made by TI company is selected as development suit. This suit includes hardware and professional software of CC2530 ZigBee Dev Kit. The program flow chart of sending data by sensor node is showed in Fig.4. For the reason that the data collected by sensor node is real-time, the setted time by timer is not long. The program flow chart of ZigBee node is showed in figure.5. Initialize CC2530, set ZigBee protocol and ZigBee protocol of numerous terminals. Estabishing router bable by searchment, every node communicates with each other and exchange router table. The program flow chart of ZigBee router is showed in Fig.6.

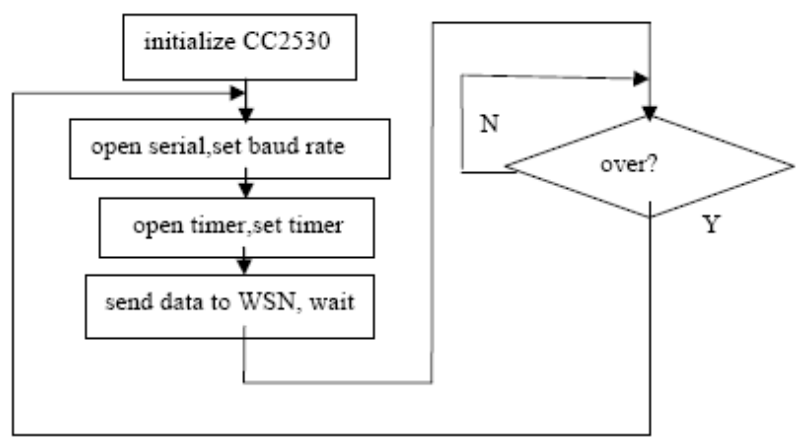

Figure 4. Program flow chart of sending data by sensor node

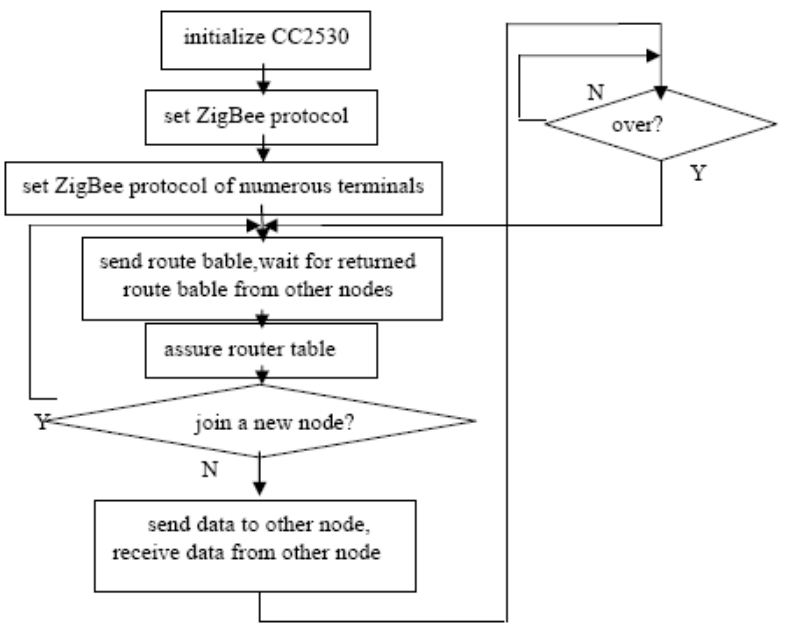

Figure 5. Program flow chart of ZigBee node

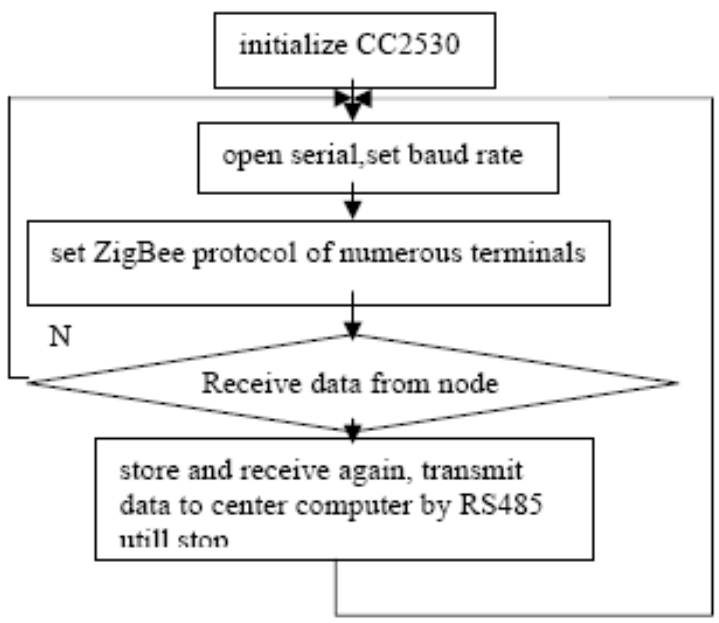

Figure 6. Program flow chart of ZigBee router

\section{V.TEST OF SYSTEM}

The test of system is to make sure the accuracy of measuring data of sensor node and trans- mision stability of wireless Ad Hoc network. Open wind turbine and observe 
the data on computer which is showed by VB program, with its data in figure.7.

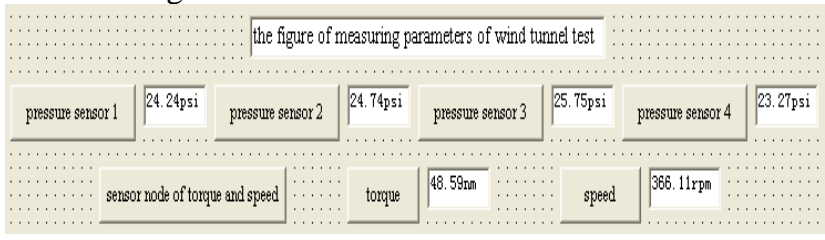

Figure 7. Test figure of the system

\section{CONCLUSION}

Confirmed by test data which is stable and reliable, the design of wireless mesurement is very useful. The paper provides hareware figures of the design and program chart of the system. Some other research need to do is testing more times for mountains of data, more data of other parameters.

\section{REFERENCES}

[1] Yu Hai-miao, Zhou Hai-zhu, Pei Xiao-mei. Environmental Value and Economic Analysis of Wind Power. Journal of Tongji University(Natural Science),37 (5), pp.708-708,2009.
[2] Ma Jian-long, Wang Jian-wen, Dong bo. Experimental modal analysis on low-frequency vibration characteristics of wind tuibine. Journal of Vibration and Shock,32(16),pp.164-170,2013.

[3] Yang hua, Xu Hao-ran, Shen Wen-zong. The method of aerodynamic experiment for a wind turbine model with the diamet. Journal of Yangzhou University(Natural Science Edition),15(4),pp.55-59,2012.

[4] Zhang Li-xun, Zhang song, Jiao Qi-fei. The method of dynamic aerodynamic experiment for Darrieus wind turbine. JOURNAL OF HUAZHONG UNIVERSITY OF SCIENCE AND TECHNOLOGY.NATURE SCIENCE,41(5),pp11-16,2013.

[5] Tang qiang, Luo Hai-tao, Zhen Xiao-yan. High Speed Wireless Network Based on Wireless Sensor Node. Journal of Detection \& Control,36(2),pp69-73,2014.

[6] Elana De Santis,Maria Domenica Di Benedetto,Maria-Gabriella Di Benedetto. Application of Hybrid Models to the Design of Ultra Wide Band Self-organizing Networks. Proceedings of the 17th international symposium on Mathematical Theory of Networks and Systems,6,pp1484-1487,2006.

[7] Jihoon Ryoo,Hwangnam Kim. Multi-sector multi-range control for self-organizing wireless networks. Journal of Network and Computer Applications,34,1848-1860,2011.

[8] A.G.Kir'yanov,A.L.Lyakhov,A.A.Safonov. A Method to Estimate Efficiency of the Connection Control Mechanisms in Wireless Self-organizing Networks. Automation and Remote Control,78(5),pp 39-56,2012.

[9] Zhi-qiang Yuan. A Design of wireless Monitoring System in Underground Mine Based on ZigBee Technology. Industrial Safety and Environmental Protection, 39(6),46-47+55,2013 